\title{
Generalized Van der Waals Equation for Liquid-Vapor Equilibria in a Stationary Gravitational Field
}

\section{Makokiyu Godlove Akumbom1, Georges Collince Fouokeng ${ }^{1 *}$, Martin Tchoffo ${ }^{1,2}$, Lukong Cornelius Fai ${ }^{1}$}

${ }^{1}$ Unité de Recherche de Matiere Condensée, d’Electronique et de Traitement de Signal (URMACETS), Department of Physics, Faculty of Science, University of Dschang, Dschang, Cameroon

${ }^{2}$ Centre d'Etudes et de Recherche en Agronomie et en Biodiversité, Faculté d'Agronomie et des Sciences Agricoles (FASA), Université de Dschang, Dschang, Cameroon

Email: ${ }^{\star}$ fouokenggc@yahoo.fr

How to cite this paper: Akumbom, M.G., Fouokeng, G.C., Tchoffo, M. and Fai, L.C. (2021) Generalized Van der Waals Equation for Liquid-Vapor Equilibria in a Stationary Gravitational Field. World Journal of Condensed Matter Physics, 11, 1-11. https://doi.org/10.4236/wjcmp.2021.111001

Received: January 9, 2021

Accepted: February 23, 2021

Published: February 26, 2021

Copyright $\odot 2021$ by author(s) and Scientific Research Publishing Inc. This work is licensed under the Creative Commons Attribution International License (CC BY 4.0).

http://creativecommons.org/licenses/by/4.0/

\begin{abstract}
The behavior of liquids undergoing phase transition in the gravitational field is studied by considering the generalized Van der Waals equation. Considering the two simple models for liquid-vapor boundary of a pure classical fluid, the generalized Van der Waals equation shows how the three critical parameters (critical temperature, critical volume and critical pressure), suffice to describe the reduced state parameters (reduced temperature, reduced volume and reduced pressure), the concentration profile and the liquid-vapor boundary position, which can be used to observe transition phenomenon. This model shows how the form of the equation can influence the vertical phase separation induced by the stationary gravitational field, and on the gas condensation effects.
\end{abstract}

\section{Keywords}

Liquid-Vapor, Phase Transition, Gravitational Field, Classical Fluid, Concentration Profile

\section{Introduction}

In recent years, many pieces of research have been made in order to investigate the effect of uniform and non-uniform gravitational fields on the perfect gas [1] [2]. That is, in order to bring out more about the field, the phase transition remains the most important and fundamental feature in thermodynamics which is considered as a link between the liquid and vapor. Towards the end of the last 
century when the extensive studies of Andrew [3] used the Van der Waals equation [4] to explain the influence of temperature and pressure on fluid's density. Thus the first theoretical description of the vapor-liquid phase transition with Van der Waals equation of State was available. The Van der Waals equation of state is useful in the scheme of generalization of the study by considering a real gas [5] [6] [7]. A generalization of this problem is the consideration of a real gas. In Refs [8] it has shown how the interesting features of a real gas and the possibility of condensation due to the existence of liquid-vapor equilibrium. Otherwise, the problem of classical liquid-vapor equilibrium has been intensively investigated in the presence of a gravitational field by using a Van der Waals equation of state especially imposing condition of equilibrium for a certain pressure at the interface, the vapor pressure $p$, that for the incompressible liquid. The critical point is characterized by fixed temperature, pressure and density and also is an example of a continuous phase transition in the case of the vapor-liquid system [9]. It is so, because the vapor-liquid critical point is one of the many critical points associated with transitions where density (as one of its specific thermodynamic properties) changes continuously in the course of transition. This approach which is valid for temperatures much lower than the critical temperature of the substance remains less general.

The influence of the constant gravitational field on the liquid-vapor equilibrium for a pure substance has been investigated and pictured as in Figure 1, where the parameters of the cell that will be used in the calculations are also defined. This pictured shows implicitly the effect of the existing constant gravitational field it is like on earth and how the denser liquid phase becomes located in the lower part of the container [10] [11]. It has been also found in Ref [10] that the absence of gravity assuming thermal equilibrium, and the liquid assumes a spherical shape by minimizing the surface energy surrounded by the vapor.

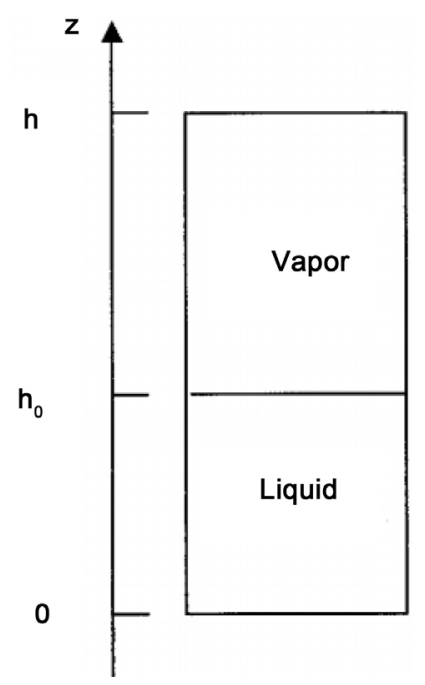

Figure 1. The cell for the study of liquid-vapor equilibrium. The cell height is $h$, and the liquid-vapor boundary is at $h_{0}$ [1]. 
Following the work done in [1], where the effect of gravity on the existence and position of the liquid-vapor boundary of a pure substance is quantitatively discussed on the basis of two simple models: a perfect gas and incompressible liquid model, and a Van der Waals fluid model, the model has been extended in this paper by considering the generalized Van der Waals equation on the existence and position of the liquid-vapor boundary of a pure substance, used in order to find the effect of gravity on a pure substance. That is, we verify the behavior of a liquid-vapor boundary in the stationary gravitational field with emphasis on the reduced parameters such as reduced pressure, reduced molar concentration and reduced temperature.

The approach used here is holistic in the sense that they involve all parameters of the system under consideration.

The organization of the paper is as follows: In Section 2, we present a brief description of the simple model that is the perfect gas and the incompressible liquid for simulation of the effect of the constant gravitational field on the pure gas system. In Section 3, the generalized Van der Walls equation is used in order to evaluate the reduced saturated pressure, the reduced temperature in terms of the reduced liquid and gas concentrations then conclude our findings in Section 4 where the theoretical results, as well as the numerical results and discussion, are treated in order to conclude in Section 5.

\section{Theoretical Study of the Perfect Gas and Incompressible Liquid in the Constant Gravitational Field}

We considered respectively a vapor-liquid as the substance in a small container and in the tall container, in order to put out in our investigation, the influence of environment.

\subsection{Environment as Small Container}

Figure 1 here represents the model of container (which is a sealed cylindrical glass tube containing a given amount of a fluid, for example $\left(\mathrm{H}_{2} \mathrm{O}, \mathrm{CO}_{2}\right.$ or plasma) used for our investigation. Considering a container of volume $V$ containing a substance of mass, $m$ that is used to determine the position of the liquid vapor interface, $h_{0}$, for a given temperature. If the height of the tube (container) is $h$, $V_{G}$ and $V_{L}$ the volumes occupied by the gas and liquid phases, respectively, then the liquid-vapor interface will be located at a height,

$$
h_{0}=f_{L} h
$$

where $f_{L}$ is the volume fraction of the liquid.

$$
f_{L}=V_{L} / V=\frac{\bar{\rho}-\rho_{G}}{\rho_{L}-\rho_{G}}
$$

with $\rho_{L}$ and $\rho_{G}$ the phase mass densities obtained from the given relation of the mass

$$
m_{L}=\rho_{L} V_{L}=m-m_{G}=m-\rho_{G}\left(V-V_{L}\right)
$$


and $\bar{\rho}=m / V$ the average density of the substance in the cell. For a perfect gas phase, the vapor mass density is given by

$$
\rho_{G}=\rho_{\text {sat }}=M P_{\text {sat }} / R T
$$

where $M$ is the molar mass and $P_{\text {sat }}$ is the vapor pressure at the given temperature $T$.

\subsection{Environment as a Tall Container}

Here, the height $\mathrm{h}$ of the container is large, the gas phase will be inhomogeneous, that is, the pressure will obey the barometric formula

$$
P(u)=P_{\text {sat }} \exp (-M g u / R T),
$$

where $g$ is the acceleration of gravity, and $u=z-h_{0}$ the height above the liquid-vapor boundary. Hence, the gas density in Equation (4) becomes

$$
\rho_{G}(u)=\rho_{\text {sat }} \exp (-M g u / R T),
$$

where $\rho_{\text {sat }}$ is given by

$$
\rho_{\text {sat }}=M P_{\text {sat }} / R T
$$

Using Equations (5), (6) and (7), the average gas density in Equation (4) can be evaluated as

$$
\overline{\rho_{G}}=\frac{\int_{h_{0}}^{h} \rho_{G}(z) \mathrm{d} z}{h-h_{0}}=\frac{P_{\text {sat }}}{g\left(h-h_{0}\right)}\left[1-\exp \left(-\frac{m g\left(h-h_{0}\right)}{R T}\right)\right]
$$

and hence Equation (1) becomes

$$
h_{0}=\frac{\bar{\rho}-\frac{P_{\text {sat }}}{g\left(h-h_{0}\right)}\left[1-\exp \left(-\frac{M g\left(h-h_{0}\right)}{R T}\right)\right]}{\rho_{L}-\frac{P_{\text {sat }}}{g\left(h-h_{0}\right)}\left[1-\exp \left(-\frac{M g\left(h-h_{0}\right)}{R T}\right)\right]}
$$

For large $h\left(h-h_{0} \rightarrow \infty\right)$, Equation (9) simplifies to

$$
h_{0}=\frac{P_{0}-P_{\text {sat }}}{g \rho_{L}}
$$

where $P_{0}=\bar{\rho} g h$ is the pressure at the bottom of the cell (That is, at $z=0$ ).

An interesting application of Equation (10) is the estimation of the effect of change in the earth's average temperature on the ocean level. From Equation (10) we have

$$
\Delta h_{0}=-\Delta P_{\text {sat }} / g \rho_{L}
$$

As it has also been shown in [1], for pure liquid water and an initial uniform temperature of $15^{\circ} \mathrm{C}$, an increase of $1^{\circ} \mathrm{C}$ implies a drop of $1 \mathrm{~cm}$ in the water level, while upon heating to $50^{\circ} \mathrm{C}, 100^{\circ} \mathrm{C}$, and $374^{\circ} \mathrm{C}$ (just below the critical point) the water level is predicted to decrease, by $1 \mathrm{~m}, 10 \mathrm{~m}$ and $2.2 \mathrm{~km}$, respectively. These calculations show that the loss of liquid water upon moderate heating is negligible in comparison to the predicted increase due to the melting of all the 
Earths' permanent ice (in the form of glaciers, ice caps, and Greenland and Antarctic ice sheets), which would cause an estimated increase of the average level of $70 \mathrm{~m}$.

A different way of changing $h_{0}$ is by modifying the acceleration of gravity, for example by means of a centrifuge. A sample initially at $g=0$ can be entirely gaseous, while once it is subjected to a gravitational field, a liquid phase may appear for extremely strong gravitational fields further phases might show up, such as one or more solid phases. The liquid phase starts to form when $g=g_{\text {min }}$ is large enough that the pressure at the bottom of the container, $P(0)$ equals the saturation pressure $P_{\text {sat }}$. Taking into account that, in the absence of a gravitational field, the pressure, $P$, and density, $\rho$ for an ideal gas are related by Equation (5) we obtain from Equation (9) an equation for $P$ and $g_{\min }$.

$$
P=P_{\text {sat }} \frac{R T}{M g_{\min } h}\left[1-\exp \left(-M g_{\min } h / R T\right)\right]
$$

For instance, to start condensing water vapor in a $h=10 \mathrm{~cm}$ cell initially at a pressure of $0.8 P_{\text {sat }}$ and at room temperature, $g_{\min }=5 \times 10 \mathrm{~m} \cdot \mathrm{s}^{-2}$.

If the container is essentially infinite $(h \rightarrow \infty)$ but the mass of the gas is finite, then Equation (12) simplifies to

$$
g_{\min }=P_{\text {sat }} / \bar{\rho} h
$$

We notice that $g_{\min }$ is proportional to the $1 / \bar{\rho}$, where $\bar{\rho}$ is the average density of the gas inside the cell for $g=0$. For the amount of water on the Earth, $\bar{\rho} \mathrm{h} \cong 3 \times 10^{6} \mathrm{~kg} \cdot \mathrm{m}^{-2}$ and using

$P_{\text {sat }}\left(15^{\circ} \mathrm{C}\right)=1.7 \times 10^{3} \mathrm{~Pa}$, one gets $g_{\text {min }}=6 \times 10^{-4} \mathrm{~m} \cdot \mathrm{s}^{-2} \ll 9.8 \mathrm{~m} \cdot \mathrm{s}^{-2}=g_{\text {Earth }}$

\section{Generalized Van der Waals Equation}

Matter irrespective of the state at which it is found is stable only over a certain range of temperatures and pressures. That is, it can move to another phase under equilibrium conditions, where the boundaries in the phase diagram delineate this behavior. The three different phases of matter can coexist in equilibrium only at the "triple point". Elsewhere only two phases can coexist i.e. Solid and Liquid along the fusion curve or Liquid and Vapor along the vaporization curve. But the vaporization curve terminates abruptly at the critical point. At this point, the temperature, pressure, and molar volume assume characteristic values $\mathrm{Tc}, \mathrm{Pc}$ and $\mathrm{Vc}$ for any given substance.

The generalized Van der Waals equation can be obtained respectively in terms of volume Equation (14) and molar concentration Equation (15) as

$$
\begin{gathered}
\left(P+\frac{a}{v^{n}}\right)(v-b)=R T \\
p=\frac{R T}{1 / C-b}-a C^{n}
\end{gathered}
$$

where $P$ is the pressure, $v$ is the molar volume. $a$ and $b$ are two different parameters characteristics of each substance. The parameter a reflects the long-range 
attractive interactions (Van der Waals forces) while the parameter $b$ reflects the short-range repulsive ones.

The Van der Waals equation is a cubic equation in the volume, which means that it has three solutions for a given pressure if the temperature, $T$, is less than the critical temperature $T_{c}$. The solutions of are the molar volume of the liquid, while the third molar volume (the third root) lying between these two has no physical meaning. If $T>T_{c}$ only one of these roots is real, while if $T=T_{c}$ the three roots merge into one. The parameters $a$ and $b$ can be related to the critical pressure $P_{c}$, the critical molar volume, $V_{c}$ and the critical temperature, $T_{c}$, Obtained by the following relations:

$$
\begin{gathered}
\frac{\partial P}{\partial V}=0 \\
\frac{\partial^{2} P}{\partial V^{2}}=0 \\
T_{C}=\frac{4 a n(n-1)^{n-1}}{K_{B} b^{n-1}(n+1)^{n+1}}, \quad V_{C}=\frac{(n+1) b}{n-1}, \quad P_{C}=\frac{a(n-1)^{n+1}}{b^{n}(n+1)^{n+1}}
\end{gathered}
$$

\section{Phase Transition Parameters}

\subsection{Theoretical Results}

In order to determine the phase transition parameters using Van der Waals equation, the hydrostatic equation has to be considered. In a gravitational field, the pressure of a fluid (gas or liquid) within a vertical cell depends on the height, $h_{0}$ obeys the balance equation,

$$
\frac{\mathrm{d}}{\mathrm{d} z} P(z)=-m g C(z)
$$

To determine reduced parameters that would help to observed phase transition, we then combine Equations (15) and (16) to obtain a generalized barometric formula in the following differential form:

$$
\frac{\mathrm{d}}{\mathrm{d} z} C(z)=-\frac{\operatorname{MgC}(\mathrm{z})}{R T /[1-b C]^{2}-n a C(z)^{n-1}}
$$

If we have assumed that the temperature is constant inside the cell and by introducing the new dimensionless variables that is the reduced gravitational energy $E_{r}=\mathrm{Mgz} /\left(R T_{c}\right)$, the reduced concentration $C_{r}=c v_{c}$, the reduced pressure $p_{r}=p / p_{c}$ and the reduced temperature $T_{r}=T / T_{c}$, in Equation (17), Equation (15) becomes

$$
p_{r}=\frac{4 n T_{r}}{(n-1)^{2}\left[\left(\frac{n+1}{n-1}\right) \frac{1}{c}-1\right]}-\left(\frac{n+1}{n-1}\right) c^{n}
$$

The concentration profile is also obtained as

$$
\frac{\mathrm{d} c}{\mathrm{~d} E_{r}}=-\frac{c}{\left(1-\left(\frac{n-1}{n+1}\right) c\right)^{2}-\frac{(n+1)^{2}}{4} c^{n-1}}
$$


Equation (19) shows that the reduced concentration profile is a function only of the reduced temperature. It is assumed that the boundary between the two phases is at the height $z=h_{0}$, so that the liquid in the cell is between $0<z \leq h_{0}$ and the gas is at $z \succ h_{0}$.

In respect of the condition that the two phases are in equilibrium when their pressures and chemical potentials are equal, from Equation (18) the following equations that describe the saturation pressure for the liquid and gaseous phases respectively:

$$
\begin{gathered}
p_{r}^{\text {sat }}=\frac{4 n T_{r}}{(n-1)^{2}\left[\left(\frac{n+1}{n-1}\right) \frac{1}{c_{L}^{*}}-1\right]}-\left(\frac{n+1}{n-1}\right) c_{L}^{{ }^{*} n} \\
p_{r}^{\text {sat }}=\frac{4 n T_{r}}{(n-1)^{2}\left[\left(\frac{n+1}{n-1}\right) \frac{1}{c_{G}^{*}}-1\right]}-\left(\frac{n+1}{n-1}\right) c_{G}^{{ }^{*} n}
\end{gathered}
$$

where $c_{L}^{*}$ and $c_{G}^{*}$ are the reduced liquid and gas concentration at the gas liquid boundary, $z=h_{0}$.

In order now to express the reduce pressure and the reduce temperature only as a function of the reduce molar concentration, we used Maxwell equal area approach.

$$
p_{r}^{\text {sat }}\left(v_{G}-v_{L}\right)=R T \ln \left(\frac{v_{G}-b}{v_{L}-b}\right)-\frac{a}{1-n}\left(v_{G}^{1-n}-v_{L}^{1-n}\right)
$$

where $V_{L}$ and $V_{G}$ are the molar volumes in the liquid and gas states at $z=h_{0}$, with $v_{L}=\left(\frac{n+1}{n-1}\right) b / c_{L}^{*}$ and $v_{G}=\left(\frac{n+1}{n-1}\right) b / c_{G}^{*}$.

In reduced variables, Equation (21) can be rewrite in the form:

$$
4 n T_{r} \ln \left(\frac{\left(\frac{n+1}{n-1}\right) \frac{1}{c_{G}}-1}{\left(\frac{n+1}{n-1}\right) \frac{1}{c_{L}}-1}\right)+(n+1)^{2}\left(c_{G}^{n-1}-c_{L}^{n-1}\right)=\left(n^{2}-1\right) p_{s a t, r}\left(\frac{1}{c_{G}}-\frac{1}{c_{L}}\right)
$$

If we combine Equation (22) with Equation (20), we obtain

$$
\ln \left(\frac{\left(\frac{n+1}{n-1}\right) \frac{1}{c_{G}}-1}{\left(\frac{n+1}{n-1}\right) \frac{1}{c_{L}}-1}\right)=\frac{\left(c_{L-} c_{G}\right)}{\left(c_{L}+c_{G}\right)}\left(\frac{n+1}{\left[(n+1)-(n-1) c_{L}\right]}+\frac{n+1}{\left[(n+1)-(n-1) c_{G}\right]}\right)
$$

From the same set of equations, the reduced saturation pressure and the reduced temperature gives

$$
p_{r}^{\text {sat }}=\frac{c_{G}^{*} c_{L}^{*}\left(c_{L}^{* n-1}-c_{G}^{* n-1}\right)\left[(n+1)-(n-1)\left(c_{L}^{*}+c_{G}^{*}\right)\right]}{(n-1)\left(c_{L}^{*}-c_{G}^{*}\right)}
$$

and 


$$
T_{r}=\frac{\left(c_{L}^{* n}-c_{G}^{*_{n}}\right)\left[(n+1)-(n-1) c_{L}^{*}\right]\left[(n+1)-(n-1) c_{G}^{*}\right]}{4 n\left(c_{L}^{*}-c_{G}^{*}\right)}
$$

\subsection{Numerical Results and Discussions}

\subsubsection{Phase Transition Observation in Terms of Reduced Pressure}

From Equation (18) the obtained reduced pressure as a function of reduced temperature is numerically investigated (see Figure 2) in order to show how the generalized form of the Van der Waals equation can be used to easy control phase transition.

Figure 2 reduced pressure as a function of reduced molar volume fraction of water is shown for a tube of $10 \mathrm{~cm}$ height and for $g=9.8 \mathrm{~m} \cdot \mathrm{s}^{-2}$ a situation that corresponds to a top reduced gravitational energy. The behavior of the curves shows that the reduced pressure increases with an increase in reduced molar volume until the corresponding critical point $\left(P_{c}=0.63\right)$ after that it decreases progressively to the infinity. The critical point that corresponds the phase transition point is observed at the reduced temperature value $T_{r}=0.9$. It is then observed that the dashed curves for vapor and the green continuous line for liquid show and intersection point at a certain reduced temperature value $T_{r}=0.9$ which a point of transition respectively from vapor to liquid and from liquid to vapor. The intersection point characterizes the supercritical point of transition after which the nature of the substance changes totally to another.

\subsubsection{Phase Transition Observation in Terms of Reduced Concentration}

Figure 3 presents the reduced concentration as a function of reduced temperature and Height in the condition that $g=9.8 \mathrm{~m} \cdot \mathrm{s}^{-2}$ that corresponds to the top reduced gravitational energy.

From Figures 3(a)-(c), it is observed that the substance changes progressively for its vapor nature to the liquid nature as the reduced temperature and the height increases. The total transition of the vapor substance to the liquid substance corresponds to the supercritical point.

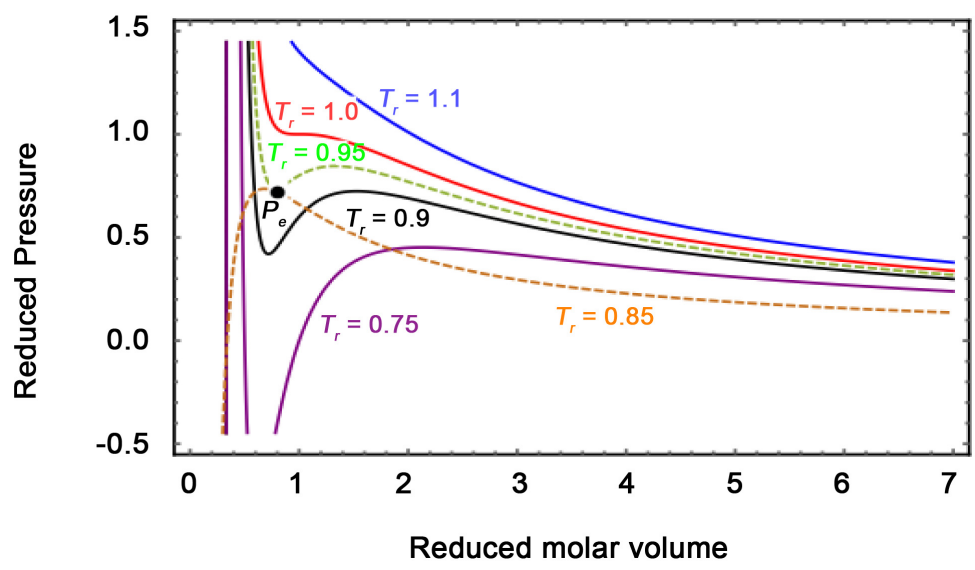

Figure 2. Reduced pressure as a function of reduced molar volume, for different values of reduced temperature. 


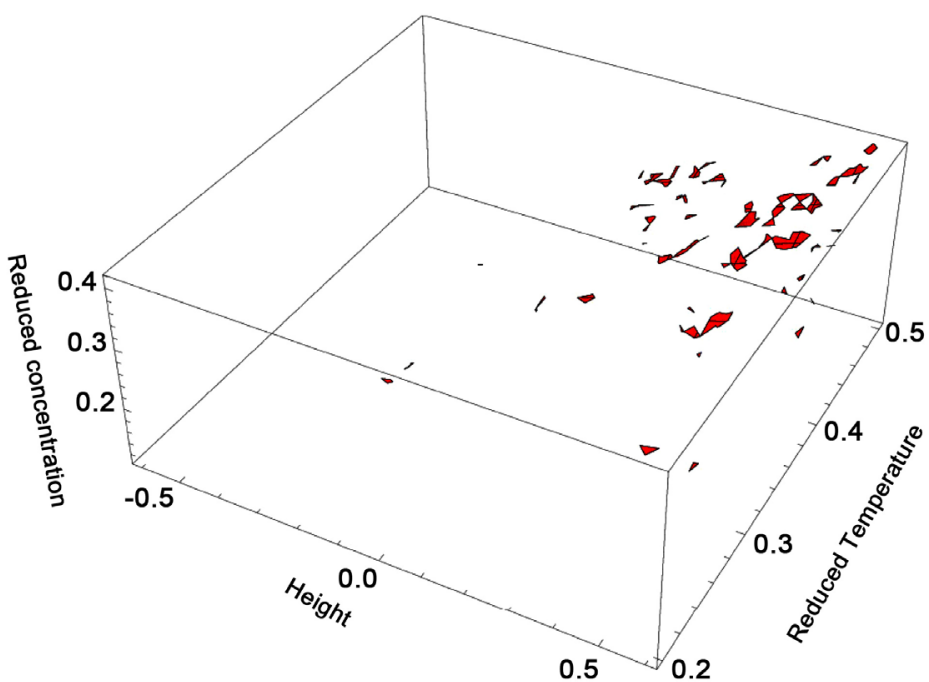

(a)

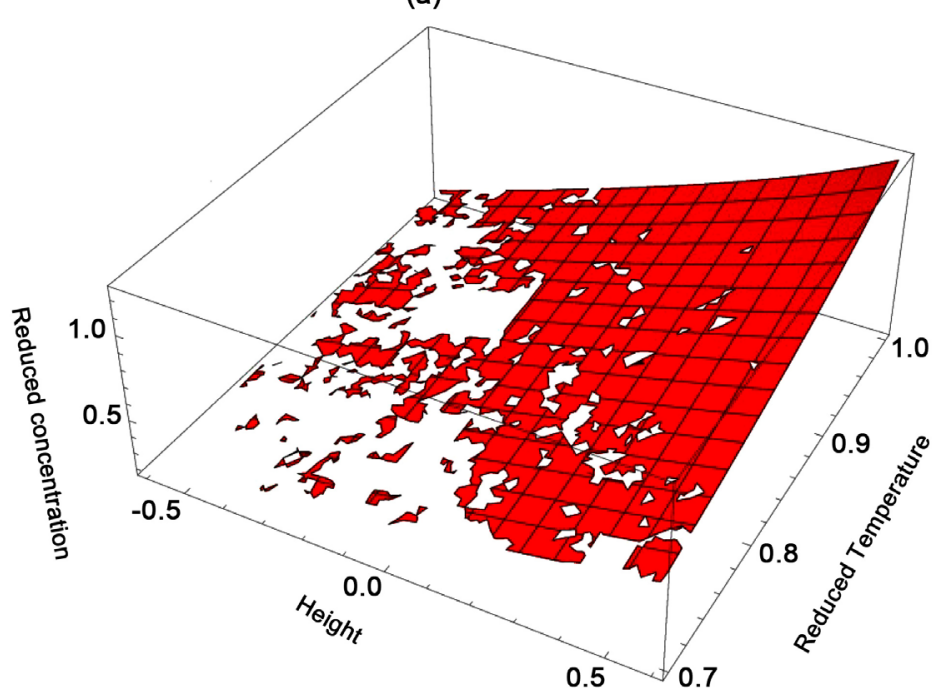

(b)

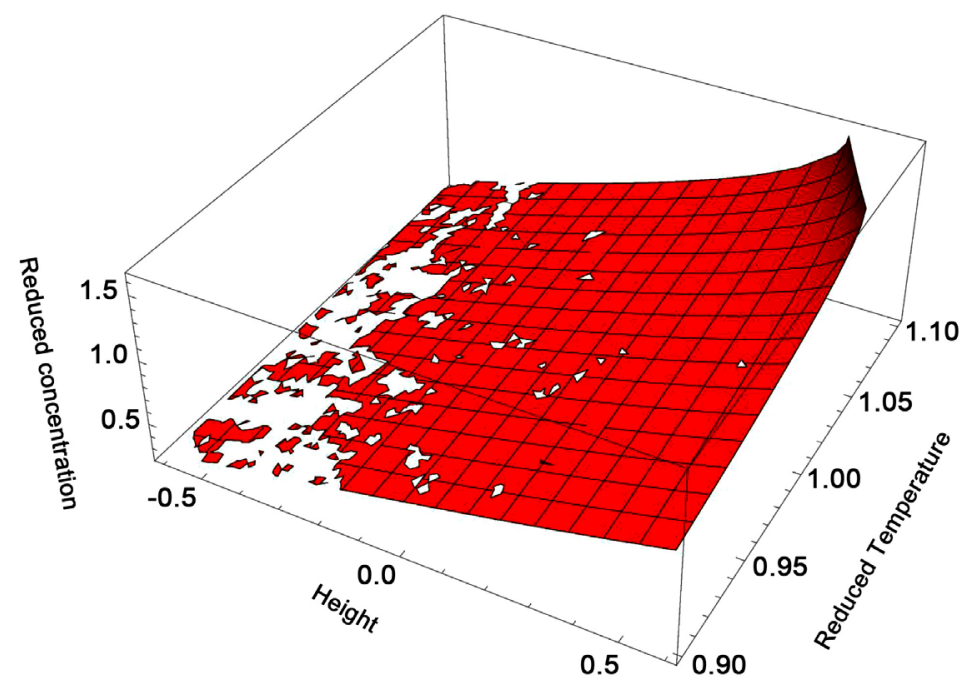

(c)

Figure 3. Reduced concentration as a function of reduced temperature and Height. 


\section{Conclusions}

In this work, the effect of gravity on the existence and position of the liquid-vapor boundary of a pure substance was quantitatively discussed on the basis of two simple models: 1) a perfect gas and incompressible liquid model, and 2) a Van der Waals fluid model. The work has been carried taking into account the generalized Van der Waal equation unlike the simple Van der Waal model treated in [1] in order to investigate the effect of the gravitational field on the liquid-vapor boundary. From the results obtained, it observed that the vapor-liquid boundary has a phase separation at the critical point defined by the critical parameters which are obtained by the first and second derivatives of the generalized Van der Waal equation of state with respect to Volume (isolation of isotherms). The first model has been shown to be a special case of the latter, for temperatures much lower than the critical temperature while for the second case (b), it has shown that the results can be obtained in terms of only two independent dimensionless parameters. The reduced temperature controls the reduced concentration vertical profile as well as the height. It is equally observed that for heights lower than $h_{o}$ (Figure 3(a)) the phenomenon of phase transition is not observed. Another interesting result of the present treatment is the possibility of directly describing the appearance of the familiar vertical phase separation in the cell. Without the introduction of a gravitational field in the laws governing the behavior of a fluid, the appearance of the phase boundary needs to be assumed. It also allows us to quantitatively discuss the conditions under which gravitational fields will induce condensation of a gas.

For future works an investigation on the phenomenon of phase separation involving a variable or position-dependent gravitational field on a vapor-liquid boundary can be carried out. Equally studies can be made to investigate the behavior of the vapor-liquid boundary using Berthelot's equation of state in the presence of different fields.

\section{Conflicts of Interest}

The authors declare no conflicts of interest regarding the publication of this paper.

\section{References}

[1] Berberan-Santos, M.N., Bodunov, E.N. and Pogliani, L. (1997) On the Barometric Formula. American Journal of Physics, 65, 404-412. https://doi.org/10.1119/1.18555

[2] Pantellini, F.G.E. (2000) A Simple Numerical Model to Simulate a Gas in a Constant Gravitational Field. American Journal of Physics, 68, 61-68. https://doi.org/10.1119/1.19374

[3] Andrews, T. (1869) On the Continuity of the Gaseous and Liquid States of Matter. Philosophical Transactions of the Royal Society, 159, 575. https://doi.org/10.1098/rstl.1869.0021

[4] Rowlinson, J.S. (1988) J.D. Van der Waals on the Continuity of the Gaseous and Liquid States. Amsterdam, North-Holland. 
[5] Kontogeorgis, G.M., Privat, R. and Jaubert, J.-N. (2019)Taking Another Look at the van der Waals Equation of State-Almost 150 Years Later. Journal of Chemical \& Engineering Data, 64, 4619-4637. https://doi.org/10.1021/acs.jced.9b00264

[6] Sokolowski-Tinten, K., Bialkowski, J., Cavalleri, A., von der Linde, D., Oparin, A., Meyer-ter-Vehn, J. and Anisimov, S.I. (1998) Transient States of Matter during Short Pulse Laser Ablation. Physical Review Letters, 81, 224-227.

https://doi.org/10.1103/PhysRevLett.81.224

[7] Basko, M.M., Krivokorytov, M.S., Yu Vinokhodov, A., Sidelnikov, Y.V., Krivtsun, V.M., Medvedev, V.V., Kim, D.A., Kompanets, V.O., Lash, A.A. and Koshelev, K.N. (2017) Fragmentation Dynamics of Liquid-Metal Droplets under Ultra-Short Laser Pulses. Laser Physics Letters, 14, Article ID: 036001.

https://doi.org/10.1088/1612-202X/aa539b

[8] El-Banbi, A. and El-Maraghi, A. (2018) PVT Property Correlations: Selection and Estimation. Gulf Professional Publishing, Houston.

[9] Jaeger, G. (1998) The Ehrenfest Classification of Phase Transitions: Introduction and Evolution. Archive for History of Exact Sciences, 53, 51-81. https://doi.org/10.1007/s004070050021

[10] Tian, J.X., Jiang, H. and Mulero, A. (2020) Equation of State for the Hard Disk Fluids. Molecular Physics, 118, e1687948. https://doi.org/10.1080/00268976.2019.1687948

[11] Hołyst, R. and Poniewierski, A. (2012) Thermodynamics for Chemists, Physicists and Engineers. Springer Science+Business Media Dordrecht, Berlin. https://doi.org/10.1007/978-94-007-2999-5 